\title{
Veillonella montpellierensis sp. nov., a novel, anaerobic, Gram-negative coccus isolated from human clinical samples
}

\author{
Estelle Jumas-Bilak, ${ }^{1}$ Jean-Philippe Carlier, $^{2}$ Hélène Jean-Pierre, ${ }^{3}$ \\ Corinne Teyssier, ${ }^{1}$ Bernard Gay, ${ }^{3}$ Josiane Campos $^{3}$ \\ and Hélène Marchandin ${ }^{3}$ \\ ${ }^{1}$ Laboratoire de Bactériologie-Virologie, Faculté de Pharmacie, 15 Avenue Charles Flahault, \\ BP 14491, 34060 Montpellier Cedex 5, France \\ ${ }^{2}$ Centre National de Référence des Bactéries Anaérobies et du Botulisme, Institut Pasteur, \\ 25 Rue du Dr Roux, 75724 Paris Cedex 15, France
${ }^{3}$ Laboratoire de Bactériologie, Hôpital Arnaud de Villeneuve, 371 Avenue du Doyen Gaston Giraud, 34295 Montpellier Cedex 5, France

Correspondence Hélène Marchandin h-marchandin@chu-montpellier.fr

\begin{abstract}
Three strains of a hitherto unknown, Gram-negative, anaerobic coccus were isolated from human samples. At the phenotypic level, the isolates displayed all the characteristics of bacteria belonging to the genus Veillonella. Sequence analysis revealed that the three strains shared $>99.5 \%$ similarity in $16 \mathrm{~S}$ rDNA sequence and $>98.4 \%$ similarity in dnaK sequence. The three unknown strains formed a separate subclade that was clearly remote from Veillonella species of human and animal origin. Based on these results, the three strains were considered to represent a novel species within the genus Veillonella, for which the name Veillonella montpellierensis is proposed.

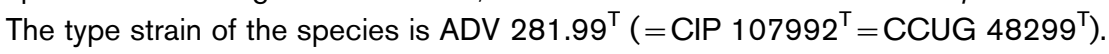

The genus Veillonella consists of small, non-fermentative, anaerobic, Gram-negative cocci. Originally classed in the family Veillonellaceae (Rogosa, 1971), the genus Veillonella was subsequently included in the family 'Acidaminococcaceae of the phylum Firmicutes (Garrity \& Holt, 2001) and the family Veillonellaceae was abolished. Serological reactions (Rogosa, 1965) and DNA-DNA hybridization studies led to the individualization of seven species in the genus Veillonella: Veillonella parvula, Veillonella atypica, Veillonella dispar, Veillonella criceti, Veillonella ratti, Veillonella rodentium and Veillonella caviae (Mays et al., 1982; Rogosa, 1984). Presently, the identification of isolates of Veillonella at the genus level is relatively straightforward, whereas the identification of Veillonella strains at the species level remains uncertain and inconvenient, owing to the lack of conventional phenotypic and biochemical discriminating tests (Kolenbrander \& Moore, 1992). Therefore, molecular methods based on 16S rDNA sequences have been used increasingly to identify Veillonella isolates (Sato et al., 1997a, b). These methods have recently been criticised, due to the low level of sequence variation among some Veillonella

Abbreviations: EM, electron microscopy; ML, maximum-likelihood; NJ, neighbour-joining.

The GenBank/EMBL/DDBJ accession number for the 16S rDNA sequence of Veillonella montpellierensis ADV $281.99^{\top} \quad(=\mathrm{CIP}$ $107992^{\top}=$ CCUG $48299^{\top}$ ) is AF473836. species, particularly between $V$. dispar and $V$. parvula, and the frequent occurrence of intra-chromosomal heterogeneity between $16 \mathrm{~S}$ rRNA gene copies in human isolates of Veillonella spp. (Marchandin et al., 2003b). Despite difficulties in species identification within the genus Veillonella, $V$. atypica, $V$. dispar and $V$. parvula are mainly reported from human flora or from clinical samples during infectious processes (Singh \& Yu, 1992; Houston et al., 1997; Liu et al., 1998; Marchandin et al., 2001). The other four species were found only in animals, except for one V. ratti isolate, strain ADV 4313.2 (=CIP 107810), which was recovered from human semen and identified after $16 \mathrm{~S}$ rDNA sequencing (GenBank accession no. AY211542) (H. Marchandin, C. Teyssier, J.-P. Carlier, E. Jumas-Bilak, M. Robert, A.-C. Artigues and H. Jean-Pierre, unpublished results).

During a survey of anaerobic, Gram-negative cocci in human clinical samples, we isolated three nitrate-reducing strains that showed a resistant phenotype when tested with a $10 \mu \mathrm{g}$ colistin disc (Rosco). The aim of the present study was to determine the taxonomic status of these isolates by using a polyphasic approach.

\section{Bacterial strains and growth conditions}

Three strains (designated ADV 281.99 ${ }^{\mathrm{T}}$, ADV 2216.03 and ADV 3198.03) were isolated from clinical samples of three 
different patients. Strain ADV 281.99 ${ }^{\mathrm{T}}$ was isolated in 1999 from gastric fluid of a newborn. It was recovered together with Gardnerella vaginalis, Escherichia coli and serotype III group B Streptococcus. Strains ADV 2216.03 and ADV 3198.03 were isolated in 2003 from amniotic fluid samples from two women (28 and 21 years old, respectively) who were hospitalized in the obstetric surgical unit of Montpellier University Hospital. Isolate ADV 2216.03 was recovered together with coagulase-negative Staphylococcus, Lactobacillus, $\alpha$-haemolytic Streptococcus and Ureaplasma urealyticum. Isolate ADV 3198.03 was recovered together with serotype III group B Streptococcus. Cultures were performed on Columbia sheep blood agar, incubated for 4 days in an anaerobic jar with the AnaeroGen system (Oxoid Unipath). The three isolates were anaerobic, Gramnegative cocci that were identified presumptively as Veillonella sp. on the basis of their capacity to reduce nitrate.

Strains ADV 281.99 , ADV 2216.03 and ADV 3198.03 were studied further in comparison with strains of each of the seven species currently described within the genus Veillonella. V. atypica ATCC $17744^{\mathrm{T}}, V$. dispar ATCC $17748^{\mathrm{T}}$, V. parvula ATCC 17745 , V. ratti ATCC $17746^{\mathrm{T}}$ and $V$. rodentium ATCC $17743^{\mathrm{T}}$ were purchased from the American Type Culture Collection (ATCC), Manassas, VA, USA. V. caviae DSM $20738^{\mathrm{T}}$ and V. criceti DSM $20734^{\mathrm{T}}$ were purchased from the Deutsche Sammlung von Mikroorganismen und Zellkulturen GmbH (DMSZ), Braunschweig, Germany, and V. parvula CIP 60.1 from the Collection de l'Institut Pasteur (CIP), Paris, France.

\section{5 rDNA analysis and phylogeny}

$16 \mathrm{~S}$ rDNA was amplified from genomic DNA as described previously (Carlier et al., 2002). The $16 \mathrm{~S}$ rDNA PCR product was sequenced directly on an Applied Biosystems automatic sequencer (Genome Express). A partial 16S rDNA sequence of $1409 \mathrm{nt}$ was determined previously for strain ADV $281.99^{\mathrm{T}}$ (GenBank accession no. AF473836; Marchandin et al., 2003a) and partial 16S rDNA sequences of 1388 and $1374 \mathrm{nt}$ were determined in this study for isolates ADV 2216.03 and ADV 3198.03, respectively. As 16S rDNA sequences of $V$. rodentium and $V$. caviae were not available, we determined almost-complete $16 \mathrm{~S}$ rDNA sequences for the type strains of these two species. GenBank accession numbers for $16 \mathrm{~S}$ rDNA sequences determined in this work are as follows: V. caviae DSM $20738^{\mathrm{T}}$, AY355140; $V$. rodentium ATCC $17743^{\mathrm{T}}$, AY514996; strain ADV 2216.03, AY355141; and strain ADV 3198.03, AY355142.

Alignment of the 16S rDNA sequences by using LALIGN software (www.expasy.ch) showed $>99.5 \%$ similarity between the sequences of strains ADV $281.99^{\mathrm{T}}$, ADV 2216.03 and ADV 3198.03. This indicated that the three isolates were closely related and could belong to the same species. BLAST analysis showed that the tested organisms were related most closely to the currently uncharacterized Veillonella sp. strain 2001-112662 (GenBank accession no. AY244769), with $99 \cdot 3-99 \cdot 6 \%$ sequence similarity. This suggested that this strain might represent the fourth member of the novel taxon. Highest similarity was found with members of the genus Veillonella, from $92.5 \%$ with $V$. criceti DSM $20734^{\mathrm{T}}$ to $94 \cdot 7 \%$ with $V$. atypica ATCC $17744^{\mathrm{T}}$. The $16 \mathrm{~S}$ rDNA sequences of strains ADV $281.99^{\mathrm{T}}$, ADV 2216.03 and ADV 3198.03 were aligned against sequences of representative strains and clones retrieved from GenBank by using the DIALIGN program (Morgenstern, 2002). The resulting $1309 \mathrm{nt}$ alignment was checked manually and used for construction of phylogenetic trees. Evolutionary trees were inferred by using the maximumlikelihood (ML) (Olsen et al., 1994), maximum-parsimony (Kluge \& Farris, 1969) and neighbour-joining (NJ) (Saitou \& Nei, 1987) methods from the PHYLIP suite of programs (Felsenstein, 1993). The F84 algorithm (Kishino \& Hasegawa, 1989) was used to generate evolutionary distance matrices for the NJ method structure; the evolutionary distance tree obtained for members of the genus Veillonella is shown in Fig. 1. Robustness of trees was evaluated by bootstrap analysis of 1000 resamplings by using the SEQBOOT and CONSENSE programs from the PHYLIP package (Felsenstein, 1993). It is evident from the 16S rDNA-based phylogeny that strains ADV $281.99^{\mathrm{T}}$, ADV 2216.03 and ADV 3198.03, together with Veillonella sp. strain 2001-112662, formed a distinct, monophyletic unit. All three treeing algorithms supported the taxonomic integrity of this clade. The monophyly of this group was supported strongly by the high bootstrap value of $100 \%$ of the ML analysis, as well as by parsimony and NJ analyses (96 and $100 \%$, respectively) (Fig. 1). A previously published phylogenetic analysis

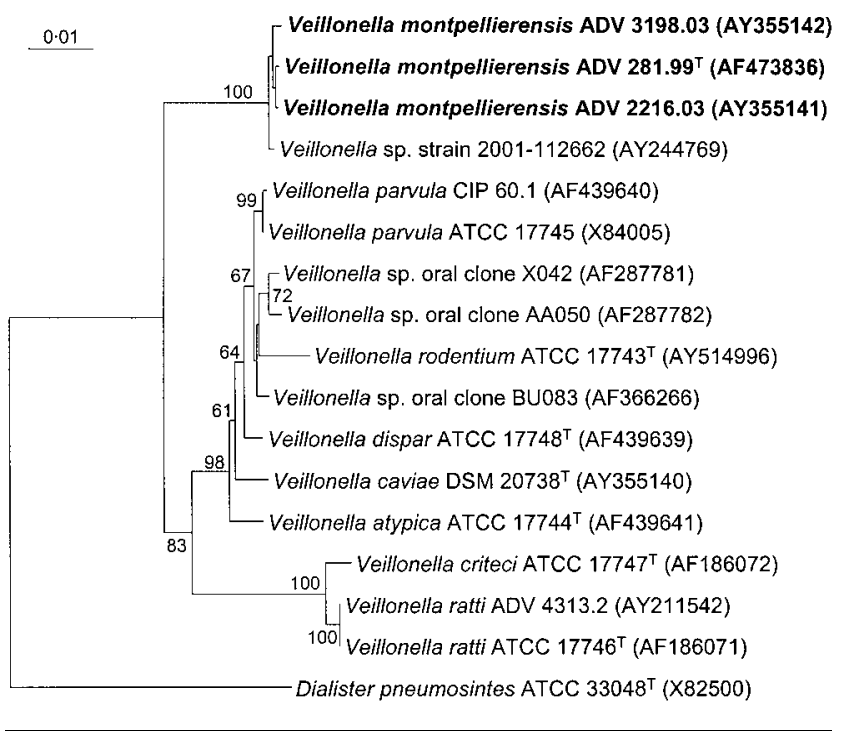

Fig. 1. Neighbour-joining tree based on nearly complete $16 \mathrm{~S}$ rDNA sequences (1309 nt), showing relationships between V. montpellierensis strains ADV $281.99^{\top}$, ADV 2216.03 and ADV 3198.03 and representatives of the genus Veillonella. Dialister pneumosintes ATCC $33048^{\top}$ was the outgroup organism. Bootstrap values higher than $60 \%$ are indicated at corresponding nodes. Bar, 0.01 substitutions per site. 
placed strain ADV $281.99^{\mathrm{T}}$ in an intermediate position among members of the genus Veillonella (Marchandin et al., 2003a). In the present study, the clade that contains strain ADV $281.99^{\mathrm{T}}$ is more deeply branched. This discordance could be explained by the sequences sampled; particularly, the sequences of $V$. caviae and $V$. rodentium and those of strains ADV 2216.03, ADV 3198.03 and Veillonella sp. 2001112662 have been included in the present trees. Nevertheless, the branching of strains ADV $281.99^{\mathrm{T}}$, ADV 2216.03, ADV 3198.03 and Veillonella sp. 2001-112662, relative to other Veillonella strains, is supported by a good bootstrap value of $83 \%$ in the NJ analysis, which is better than that obtained in the previous tree (67\%), and by the congruence observed between the three phylogenetic methods.

$16 \mathrm{~S}$ rDNA sequence similarity values of strains ADV $281.99^{\mathrm{T}}$, ADV 2216.03 and ADV 3198.03 with other members of the genus Veillonella are appropriate for determining intrageneric relationships for species definition (Stackebrandt \& Goebel, 1994) and indicate that these three strains represent a novel species within the genus Veillonella. However, recent results showed that $16 \mathrm{~S}$ rDNA-based approaches were not suitable for the identification of $V$. dispar and $V$. parvula, owing to the close genetic relationship observed for these two species and to the relatively high level of intra-chromosomal heterogeneity between the four $16 \mathrm{~S}$ rRNA gene copies observed in human isolates of Veillonella spp. Furthermore, this heterogeneity impaired the phylogenetic placement of a clinical strain in the tree (Marchandin et al., 2003b). As a consequence and despite the robustness of the $16 \mathrm{~S}$ rDNA phylogeny we obtained here, we chose to analyse another genetic marker.

\section{dnaK sequence analysis and phylogeny}

The $70 \mathrm{kDa}$ heat-shock protein gene (dnaK) was generally present as one copy in bacterial genomes and showed higher interspecies variability than the $16 \mathrm{~S}$ rRNA gene. These two characteristics should avoid the pitfalls of the 16S rDNAbased taxonomy of the genus Veillonella. A 700 bp dnaK fragment was amplified and sequenced as described previously (Marchandin et al., 2003b) for strains ADV
281.99 ${ }^{\mathrm{T}}$, ADV 2216.03, ADV 3198.03, V. caviae DSM $20738^{\mathrm{T}}$, V. criceti DSM $20734^{\mathrm{T}}$, V. parvula ATCC 17745 , $V$. ratti ATCC $17746^{\mathrm{T}}$ and $V$. rodentium ATCC $17743^{\mathrm{T}}$. Sequences were deposited in GenBank under accession numbers AY353717, AY353715, AY353716, AY353714, AY353713, AY514999, AY514998 and AY514997, respectively. Sequence data obtained were aligned with those of V. atypica ATCC $17744^{\mathrm{T}}, V$. dispar ATCC $17748^{\mathrm{T}}$ and V. parvula CIP 60.1 (previously deposited in databases) and were submitted to phylogenetic analysis, as described above. Phylogenetic trees obtained by analysis of $396 \mathrm{nt}$ revealed that the overall topology was congruent with that of $16 \mathrm{~S}$ rDNA-based trees except for the relative branching of $V$. caviae, which was supported by a very low bootstrap value in the $d n a K$ tree. The three strains described here formed a distinct lineage, supported by a high bootstrap value, within the genus Veillonella (Fig. 2). Pairwise similarity analysis showed low levels of dnaK sequence similarity between strain ADV $281.99^{\mathrm{T}}$ and other Veillonella species, from $79 \%$ with $V$. ratti ATCC $177346^{\mathrm{T}}$ to $86 \cdot 4 \%$ with $V$. dispar ATCC $17748^{\mathrm{T}}$, whereas strains ADV $281.99^{\mathrm{T}}$ and ADV 2216.03 were identical in $d n a K$ sequence. The $d n a K$ sequence of strain ADV 3198.03 shared only $98.4 \%$ similarity with those of strains ADV 281.99 $9^{\mathrm{T}}$ and ADV 2216.03. Regarding the sequence similarity level of $99.7 \%$ between the two strains of $V$. parvula, the affiliation of the three strains in a single species is questionable. However, as no higher than $96 \%$ similarity was observed between $d n a K$ sequences of the type strains of the related species $V$. parvula and $V$. dispar, inclusion of strain ADV 3198.03 in the same species as strains ADV $281.99^{\mathrm{T}}$ and ADV 2216.03 appeared reasonable.

Finally, molecular and phylogenetic data obtained for $16 \mathrm{~S}$ rDNA and dnaK sequences were congruent and suggested that the three clinical strains characterized herein represent a novel species within the genus Veillonella. A more complete phenotypic analysis of the strains was undertaken.

\section{Colony and cell morphology}

The three strains grew on Columbia blood agar at $37^{\circ} \mathrm{C}$ under anaerobic conditions, forming small colonies of

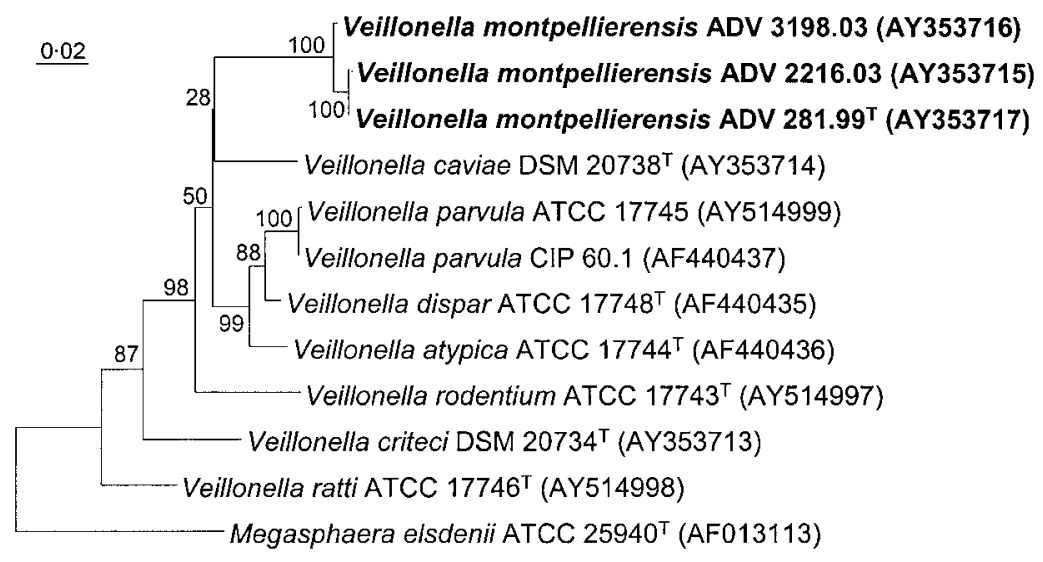

Fig. 2. Neighbour-joining tree based on partial dnaK sequences (396 nt), showing relationships between $V$. montpellierensis strains ADV 281.99 ${ }^{\top}$, ADV 2216.03 and ADV 3198.03 and representatives of the genus Veillonella. Megasphaera elsdenii ATCC $25940^{\top}$ was the outgroup organism. Numbers at nodes indicate percentages of bootstrap support, based on analysis of 1000 resampled datasets. Bar, 0.02 substitutions per site. 
1-3 mm diameter, which were smooth, opaque and greyishwhite. The three isolates were Gram-negative, non-motile, non-sporulating, tiny cocci that were spherical or slightly elongated and were organized singly, in pairs or occasionally in short chains. General morphology of the cells was observed after negative staining as described previously (Marchandin et al., 2003a), confirming that the cells were spherical, either single or in short chains of two to four cells. Most cells were $0 \cdot 3-0 \cdot 5 \mu \mathrm{m}$ in diameter. The convoluted nature of the cell surface and the diplococcal shape of the cells were observed (Fig. 3a) and were in accordance with the observations of Bladen \& Mergenhagen (1964) for other Veillonella species. Ultrastructure of thin sections was also observed by electron microscopy (EM) (Fig. 3b) and revealed typical Gram-negative surface layers, which consisted of an outer membrane, a thin peptidoglycan layer and a convoluted cytoplasmic membrane (Fig. 3c), as described previously for several representatives of the family 'Acidaminococacceae', e.g. Veillonella spp. (Bladen \& Mergenhagen, 1964), Megasphaera spp. (Marchandin et al., 2003a), Selenomonas ruminantium (Kamio \& Takahashi, 1980; Kalmokoff et al., 2000) and Centipeda periodontii (Males et al., 1984).

\section{Biochemical characteristics}

The three strains displayed susceptibility to discs that contained $1 \mathrm{mg}$ kanamycin or $4 \mu \mathrm{g}$ metronidazole and resistance to discs that contained $5 \mu \mathrm{g}$ vancomycin or $10 \mu \mathrm{g}$ colistin (Rosco). These characteristics were also observed for V. ratti (strain ATCC $17746^{\mathrm{T}}$ and clinical strain ADV 4313.2), whereas strains of the other six Veillonella species were found to be susceptible to the $10 \mu \mathrm{g}$ colistin disc. Further characterization by conventional biochemical tests (Holdeman et al., 1977) and determination of metabolic end products (Carlier, 1985) were performed. The main phenotypic characteristics corresponded to those of the genus Veillonella as described by Rogosa (1971). Gas production was noted. Presence of catalase was strain-dependent, as only one of the three strains displayed catalase activity. Urease and cytochrome oxidase activities were not detected. Gelatin was not liquefied and milk was not modified. Indole and desulfoviridin were not produced. Aesculin was not hydrolysed. Lactate and succinate were fermented to propionate. Acid was not produced from aesculin, arabinose, cellobiose, galactose, glucose, glycerol, inositol, inulin, lactose, maltose, mannitol, mannose, melezitose, melibiose, raffinose, rhamnose, ribose, sucrose, salicin, sorbitol, trehalose or xylose. The major metabolic end products in TGY broth (Carlier et al., 2002) were acetic and propionic acids $\left(8.5\right.$ and $6.9 \mathrm{mmol} \mathrm{l}^{-1}$, respectively, for strain ADV $281.99^{\mathrm{T}}$, $5 \cdot 5$ and $3 \cdot 6 \mathrm{mmol}^{-1}$ for strain ADV 2216.03 and $5 \cdot 5$ and $4 \cdot 6 \mathrm{mmol} \mathrm{l}^{-1}$ for strain ADV 3198.03).

\section{Cellular fatty acid analysis}

Cellular fatty acid composition was analysed by GC according to Veys et al. (1989). Briefly, strains were grown anaerobically in $10 \mathrm{ml}$ TGYH (Carlier et al., 2002) for 48$72 \mathrm{~h}$ and methyl esters were chromatographed on a fusedsilica capillary column $(25 \mathrm{~m} \times 0.25 \mathrm{~mm}$ ID) coated with $5 \%$ methyl phenyl silicone. The cellular fatty acid composition of the three clinical strains was very similar to that of other species of the genus Veillonella (Table 1). It is also noteworthy that all species studied contained a unidentified compound, which eluted between 17:0 iso and $\Delta$-cis-9, 10methylenehexadecanoate $(17: 0 \Delta$-cyclic 9, 10) (Table 1). With the exception of $V$. caviae DSM $20738^{\mathrm{T}}$, the only notable difference with the other type strains consisted of a smaller percentage of 13:0 and a higher amount of cis-9octadecenoic acid $(18: 1 \omega 9 \mathrm{cis})$. Also, levels of $18: 0$ that were observed for these new isolates tended to be slightly higher than those obtained for the other species.

Notwithstanding the absence of clearly distinct phenotypic characteristics between the different species of the genus Veillonella, it is clear from the genotypic data that strains ADV 281.99 ${ }^{\mathrm{T}}$, ADV 2216.03, ADV 3198.03 and probably Veillonella sp. 2001-112662 are members of a novel species of the genus Veillonella. It is therefore proposed that these strains should be classified in the genus Veillonella as Veillonella montpellierensis sp. nov. Considering that the three strains in our study were the only anaerobic bacteria that were recovered from the three corresponding clinical
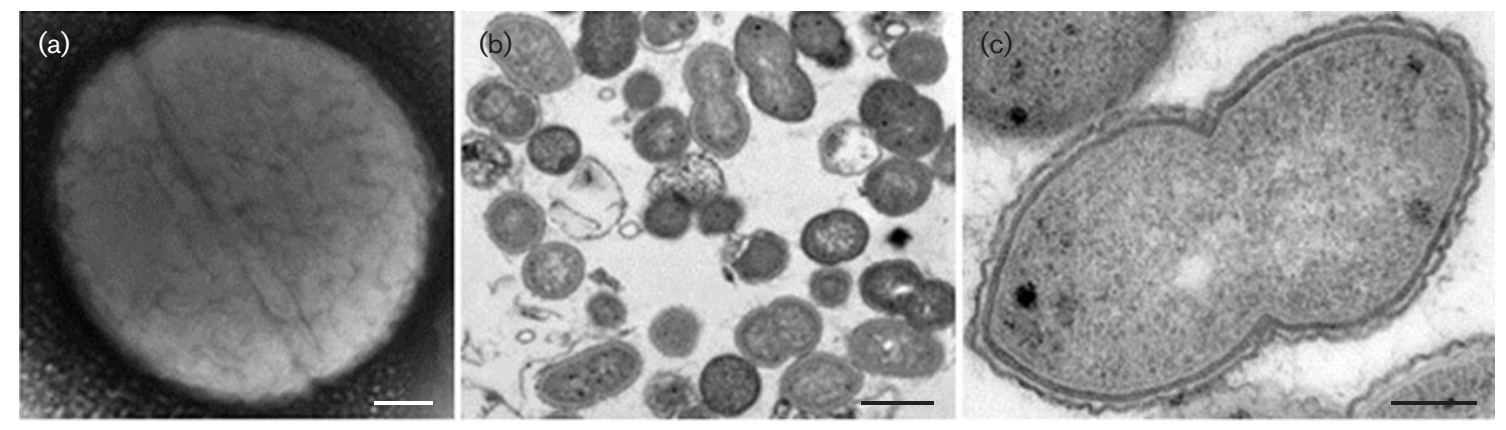

Fig. 3. Ultrastructure of strain ADV $281.99^{\top}$. (a) General morphology after negative staining; (b) general morphology after EM of ultrathin sections; (c) cell wall and membranes after EM of ultrathin sections. Bars: (a) $166 \mathrm{~nm}$; (b) $666 \mathrm{~nm}$; (c) $200 \mathrm{~nm}$. 
Table 1. Relative content (\%) of cellular fatty acids of V. montpellierensis and other Veillonella species

Reference strains: 1, V. atypica ATCC $17744^{\mathrm{T}} ; 2$, V. caviae DSM $20738^{\mathrm{T}} ; 3$, V. criceti DSM $20734^{\mathrm{T}} ; 4$, V. dispar ATCC $17748^{\mathrm{T}}$; 5 , V. ratti ATCC $17746^{\mathrm{T}} ; 6$, V. rodentium ATCC $17743^{\mathrm{T}} ; 7, V$. parvula ATCC 17745 . Fatty acid nomenclature: unsaturated fatty acids, the position of the double bond can be located by counting from the methyl $(\omega)$ end of the carbon chain; cis and trans isomers are indicated by the suffixes $c$ and $t$, respectively.

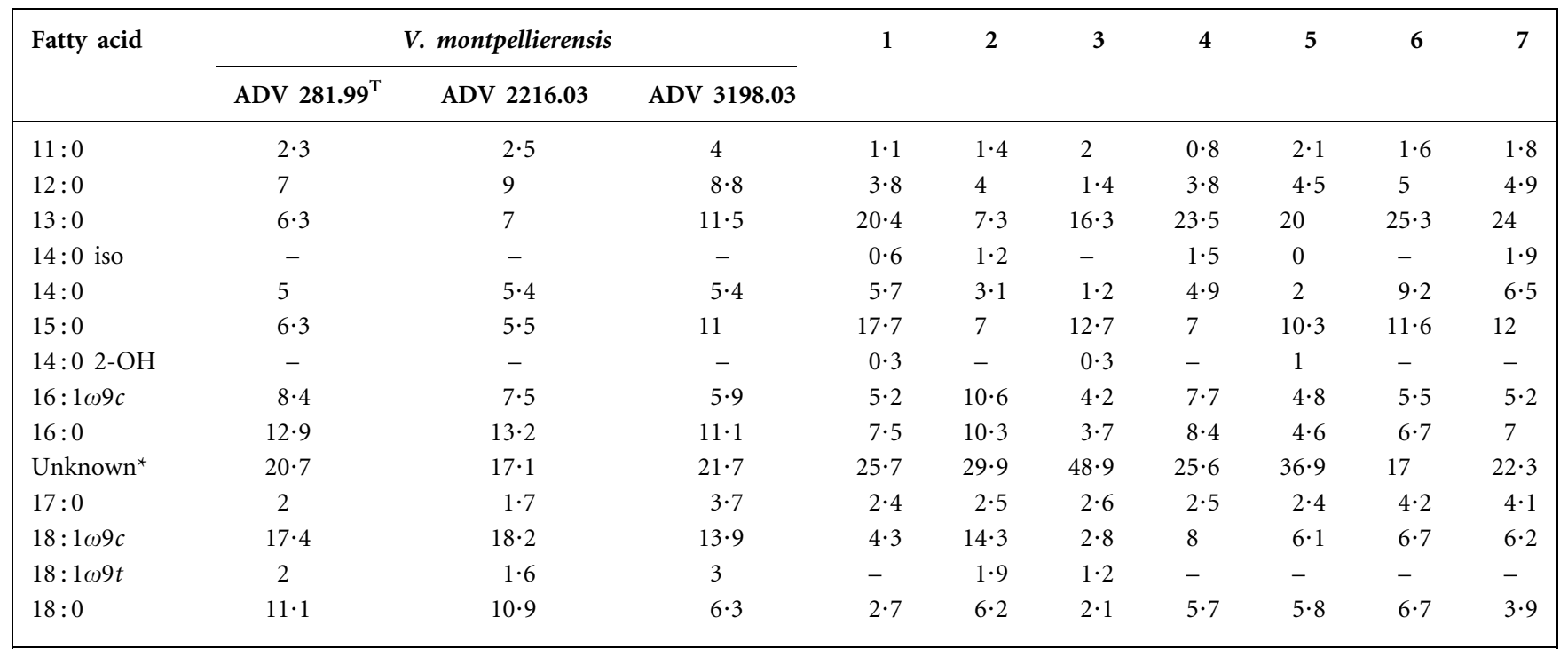

${ }^{\star}$ Unknown compound that eluted between 17:0 iso and 17:0 $\Delta$ (cyclic 9, 10).

samples and that Veillonella sp. strain 2001-112662 was isolated from the blood culture of a female patient who presented with septic shock, the novel species may have pathogenic potential.

\section{Description of Veillonella montpellierensis sp. nov.}

Veillonella montpellierensis (mont.pel.li.er.en'sis. N.L. fem. adj. montpellierensis pertaining to Montpellier in the south of France, where the type strain and two other strains supporting the description of the species were isolated).

Cells are coccoid $(0.3-0.5 \mu \mathrm{m}$ in diameter) and occur singly, in pairs or in short chains. Gram-negative, nonmotile, non-sporulating and with a convoluted surface. Colonies on Columbia blood agar are 1-3 $\mathrm{mm}$ in diameter and appear smooth, opaque and greyish-white. Strictly anaerobic and oxidase-negative. Nitrate is reduced. Gas is produced. Major metabolic end products are acetic and propionic acids. Major cellular fatty acids are 12:0, 13:0, $14: 0,15: 0,16: 1 \omega 9$ cis, $16: 0,18: 1 \omega 9$ cis and $18: 0$. Strains contained an unidentified compound, which eluted between $17: 0$ iso and $\Delta$-cis-9, 10-methylenehexadecanoate (17:0 $\Delta$-cyclic 9,10$)$. Can be differentiated from other species of the genus Veillonella by $16 \mathrm{~S}$ rDNA and dnaK sequencing.

The type strain is ADV $281.99^{\mathrm{T}}\left(=\mathrm{CIP} 107992^{\mathrm{T}}=\mathrm{CCUG}\right.$ $48299^{\mathrm{T}}$ ) and reference strains are ADV 2216.03 and ADV 3198.03. Found in human clinical samples.

\section{Acknowledgements}

We are indebted to J. P. Euzéby for helpful comments on nomenclatural issues. We thank A.-C. Artigues, M. Bedora, E. Cazalet, I. Diego, S. Larose and M. Manich for excellent technical assistance. This work is partly supported by the association ADEREMPHA, Montpellier, France.

\section{References}

Bladen, H. A. \& Mergenhagen, S. E. (1964). Ultrastructures of Veillonella and morphological correlation of an outer membrane with particles associated with endotoxic activity. J Bacteriol 88, 1482-1492.

Carlier, J.-P. (1985). Gas chromatography of fermentation products: its application in diagnosis of anaerobic bacteria. Bull Inst Pasteur 83, 57-69.

Carlier, J.-P., Marchandin, H., Jumas-Bilak, E., Lorin, V., Henry, C., Carrière, C. \& Jean-Pierre, H. (2002). Anaeroglobus geminatus gen. nov., sp. nov., a novel member of the family Veillonellaceae. Int J Syst Evol Microbiol 52, 983-986.

Felsenstein, J. (1993). PHYLIP (phylogeny inference package), version 3.5c. Department of Genetics, University of Washington, Seattle, USA.

Garrity, G. M. \& Holt, J. G. (2001). Taxonomic outline of the Archaea and Bacteria. In Bergey's Manual of Systematic Bacteriology, 2nd edn, vol. 1, pp. 155-166. Edited by D. R. Boone \& R. W. Castenholz. New York: Springer.

Holdeman, L. V., Cato, E. P. \& Moore, W. E. C. (editors) (1977). Anaerobe Laboratory Manual, 4th edn. Blacksburg, VA: Virginia Polytechnic Institute and State University. 
Houston, S., Taylor, D. \& Rennie, R. (1997). Prosthetic valve endocarditis due to Veillonella dispar: successful medical treatment following penicillin desensitization. Clin Infect Dis 24, 1013-1014.

Kalmokoff, M. L., Austin, J. W., Whitford, M. F. \& Teather, R. M. (2000). Characterization of a major envelope protein from the rumen anaerobe Selenomonas ruminantium OB268. Can J Microbiol 46, 295-303.

Kamio, Y. \& Takahashi, H. (1980). Outer membrane proteins and cell surface structure of Selenomonas ruminantium. J Bacteriol 141, 899-907.

Kishino, H. \& Hasegawa, M. (1989). Evaluation of the maximum likelihood estimate of the evolutionary tree topologies from DNA sequence data, and the branching order in Hominoidea. J Mol Evol 29, $170-179$.

Kluge, A. G. \& Farris, F. S. (1969). Quantitative phyletics and the evolution of anurans. Syst Zool 18, 1-32.

Kolenbrander, P. E. \& Moore, L. V. H. (1992). The genus Veillonella. In The Prokaryotes, 2nd edn, pp. 2034-2047. Edited by H. G. Balows, M. Trüper, W. Dworkin, W. Harder \& K.-H. Schleifer. New York: Springer.

Liu, J. W., Wu, J. J., Wang, L. R., Teng, L. J. \& Huang, T. C. (1998). Two fatal cases of Veillonella bacteremia. Eur J Clin Microbiol Infect Dis 17, 62-64.

Males, B. M., Berthold, P., Dougherty, P. A. \& Listgarten, M. A. (1984). Helical flagellation in Centipeda periodontii, a Gram-negative, anaerobic bacillus from periodontitis lesions. J Gen Microbiol 130, 185-191.

Marchandin, H., Jean-Pierre, H., Carrière, C., Canovas, F., Darbas, H. \& Jumas-Bilak, E. (2001). Prosthetic joint infection due to Veillonella dispar. Eur J Clin Microbiol Infect Dis 20, 340-342.

Marchandin, H., Jumas-Bilak, E., Gay, B., Teyssier, C., Jean-Pierre, H., Siméon de Buochberg, M., Carrière, C. \& Carlier, J.-P. (2003a). Phylogenetic analysis of some Sporomusa sub-branch members isolated from human clinical specimens: description of Megasphaera micronuciformis sp. nov. Int J Syst Evol Microbiol 53, 547-553.

Marchandin, H., Teyssier, C., Siméon de Buochberg, M., Jean-Pierre, H., Carrière, C. \& Jumas-Bilak, E. (2003b). Intra-chromosomal heterogeneity between the four $16 \mathrm{~S}$ rRNA gene copies in the genus Veillonella: implications for phylogeny and taxonomy. Microbiology 149, 1493-1501.
Mays, T. D., Holdeman, L. V., Moore, W. E. C., Rogosa, M. \& Johnson, J. L. (1982). Taxonomy of the genus Veillonella Prévot. Int J Syst Bacteriol 32, 28-36.

Morgenstern, B. (2002). A simple and space-efficient fragmentchaining algorithm for alignment of DNA and protein sequences. Appl Math Lett 15, 11-16.

Olsen, G. J., Matsuda, H., Hagstrom, R. \& Overbeek, R. (1994). fastDNAml: a tool for construction of phylogenetic trees of DNA sequences using maximum likelihood. Comput Appl Biosci 10, 41-48.

Rogosa, M. (1965). The genus Veillonella. IV. Serological groupings, and genus and species emendations. J Bacteriol 90, 704-709.

Rogosa, M. (1971). Transfer of Veillonella Prévot and Acidaminococcus Rogosa from Neisseriaceae to Veillonellaceae fam. nov., and the inclusion of Megasphaera Rogosa in Veillonellaceae. Int J Syst Bacteriol 21, 231-233.

Rogosa, M. (1984). Anaerobic Gram-negative cocci. In Bergey's Manual of Systematic Bacteriology, vol. 1, pp. 680-685. Edited by N. R. Krieg \& J. G. Holt. Baltimore: Williams \& Wilkins.

Saitou, N. \& Nei, M. (1987). The neighbor-joining method: a new method for reconstructing phylogenetic trees. Mol Biol Evol 4 , 406-425.

Sato, T., Matsuyama, J., Sato, M. \& Hoshino, E. (1997a) Differentiation of Veillonella atypica, Veillonella dispar and Veillonella parvula using restricted fragment-length polymorphism analysis of $16 \mathrm{~S}$ rDNA amplified by polymerase chain reaction. Oral Microbiol Immunol 12, 350-353.

Sato, T., Sato, M., Matsuyama, J. \& Hoshino, E. (1997b). PCRrestriction fragment length polymorphism analysis of genes coding for 16S rRNA in Veillonella spp. Int J Syst Bacteriol 47, 1268-1270.

Singh, N. \& Yu, V. L. (1992). Osteomyelitis due to Veillonella parvula: case report and review. Clin Infect Dis 14, 361-363.

Stackebrandt, E. \& Goebel, B. M. (1994). Taxonomic note: a place for DNA-DNA reassociation and 16S rRNA sequence analysis in the present species definition in bacteriology. Int J Syst Bacteriol 44, 846-849.

Veys, A., Callewaert, W., Waelkens, E. \& Van den Abbeele, K. (1989). Application of gas-liquid chromatography to the routine identification of nonfermenting gram-negative bacteria in clinical specimens. J Clin Microbiol 27, 1538-1542. 